\title{
Preeclampsia-an immune disease? An epidemiologic narrative
}

\author{
Gustaaf Albert Dekker ${ }^{*}{ }^{\circledR}$, Pierre Yves Robillard ${ }^{2,3}[0$ \\ ${ }^{1}$ University of Adelaide, Women's and Children's Division Northern Adelaide Local Health Network Elizabeth Vale, SA 5112, \\ Australia \\ ${ }^{2}$ Neonatology, Centre Hospitalier Universitaire Sud-Reunion, BP 350, 97448 Saint-Pierre cedex, Reunion, France \\ ${ }^{3}$ Centre d'études périnatales Océan-Indien (CEPOI). Centre Hospitalier Universitaire Sud-Reunion, BP 350, 97448, Saint-Pierre \\ cedex, Reunion, France
}

*Correspondence: Gustaaf Albert Dekker, University of Adelaide, Women's and Children's Division Northern Adelaide LocalHealth Network Elizabeth Vale, SA 5112, Australia. gustaaf.dekker@adelaide.edu.au

Academic Editor: Satish Kumar Gupta, Indian Council of Medical Research, India

Received: July 12, 2021 Accepted: November 15, 2021 Published: December 31, 2021

Cite this article: Dekker GA, Robillard PY. Preeclampsia—an immune disease? An epidemiologic narrative. Explor Immunol. 2021;1:325-40. https://doi.org/10.37349/ei.2021.00022

\begin{abstract}
The maternal syndrome preeclampsia is triggered by syncytiotrophoblast (STB) stress; the heterogeneity of the syndrome is caused by the different pathways leading to this STB stress. Inflammation plays a pivotal role in the pathogenesis of preeclampsia. While, the immune system at large is therefore intimately involved in the causation of this heterogeneous syndrome, the role of the adaptive immune system is more controversial. The classic paradigm placed preeclampsia as the disease of the nulliparous pregnant women. Up to the later part of the 20th century, human reproduction, particularly in Western societies, was characterised by a low rate of pre-marital sex, and the great majority of children being born within one stable sexual relationship. More prolonged periods of regular sexual intercourse within a stable relationship have been demonstrated to reduce the risk of preeclampsia and fetal growth restriction. Primarily animal studies have indeed shown that repetitive sperm exposure leads to partner specific mucosal tolerance. Societal changes made partner change over the reproductive period of individual women extremely common. For the adaptive immune system of multiparous women, being pregnant in a new sexual relationship (primipaternity) would represent being faced with a new "hemi-allograft". In these pregnancies, potential couple-specific immune "maladaptation" could lead to the superficial cytotrophoblast invasion of the spiral arteries, known to be associated with early-onset preeclampsia. Having a new pregnancy in a different relationship does indeed increase the risk for this type of preeclampsia. Large epidemiologic population studies identified prolonged birth interval but not "primipaternity" as a risk factor for preeclampsia in multiparous women. This apparent contradiction is explained by the fact that the great majority of preeclampsia cases in these population studies involve term preeclampsia. In late-onset preeclampsia, the far more common phenotype of the syndrome, STB stress is not caused by lack of proper spiral artery modification, but involves maternal genetic predisposition to cardiovascular and metabolic disease, with in particular obesity/metabolic syndrome representing major players. Partner or couple specific issues are not detectable in this disease phenotype.
\end{abstract}




\section{Keywords}

Preeclampsia heterogeneous syndrome, adaptive immune system, early-onset preeclampsia

\section{Introduction}

Preeclampsia-an immune disease? This appears a simple and clear question. But, as most preeclampsia researchers will agree-nothing about preeclampsia is truly "simple". Also, the term "immune disease" can be approached or interpreted in many ways. If the term "immune disease" would indicate any involvement of the immune system "at large", i.e., including the broad biological concept of inflammation, the innate and adaptive immune system the answer to the question would be a resounding "yes". Inflammation is clearly a central feature in the pathogenesis of the preeclampsia syndrome [1]. However, when addressing the question "preeclampsia-an immune disease", most clinical and epidemiological researchers since the early reports in the 1980's focussed on partner specificity and as such the adaptive immune system. Using this narrower approach, the data are more conflicting. In hindsight, the lack of consistency in research data on aspects like partner change and the length of sexual relationship are not surprising. Over the last 10-15 years, Redman and his Oxford collaborators [2] repeatedly warned the preeclampsia researchers not to approach preeclampsia as a singular entity but as a heterogeneous syndrome. Belated, but now progressively, researchers have learned to heed his message. Particular progress has been made in understanding the pathogenesis of the maternal aspects of the syndrome, the systemic endothelial cell dysfunction. Redman et al. [3] introduced the term syncytiotrophoblast (STB) stress and made important observations on the senescence of the STB. The STB, being a syncytium, cannot repair itself and ages in contrast to for instance liver parenchymal cells. The "aging" STB gets progressively stressed; inflammation is an integral part of the "aging" process. The stressed STB releases a multitude of factors, including various inflammatory cytokines and importantly the anti-angiogenic factors soluble fms-like tyrosine kinase-1 (sFlt-1, positive stress signal), while (negative stress signal) the STB release of placental growth factor (PLGF), a pro-angiogenic factor drops. Even uncomplicated term pregnancies demonstrate a degree of "physiologic" STB stress demonstrated by the normal drop in PLGF and increase in sFlt-1 occurring after 30-32 weeks [3]. One might argue that this "physiological" imbalance in term normal pregnancies explains the physiologic increase in blood pressure, to a level close to the booking blood pressure, typically seen in the late 3rd trimester.

There is now consensus that there are at least 2 main subtypes, early-onset preeclampsia (preeclampsia leading to mostly iatrogenic preterm birth prior to 34 week's gestation) and late-onset preeclampsia (birth > 34 week's gestation). Early-onset preeclampsia is typically linked to the classic shallow, endovascular cytotrophoblast (CTB) invasion in the spiral arteries [3]. Initially these non-transformed spiral arteries lead to pulsatile high velocity flow patterns damaging the vulnerable STB [4]. Later on, ischemia-reperfusion injury further leads to progressive and severe STB stress. Typically, these cases also demonstrate the fetal phenotype, i.e., intra-uterine growth restriction (IUGR). Proper couple specific immune interactions are clearly of pivotal importance in allowing the appropriately timed and progressive endovascular CTB invasion in the spiral arteries, and disturbances at this level (immune maladaptation) are expected to be associated with early-onset disease. While late-onset preeclampsia centres around interactions between STB senescence and a maternal genetic predisposition to cardiovascular and metabolic disease [4]. Obesity and other variables making up the metabolic syndrome certainly represent some of the major players [5, 6], with even gestational weight gain during pregnancy representing an important factor [7]. Inflammation, a key STB stressor, plays a major role in late-onset preeclampsia, while partner specific issues are mostly not discernible. Obviously, the binary split early-versus late-onset preeclampsia is more or less artificial. The balance between "placental" and "maternal" causations or risk factors varies among individual women, and couples across the spectrum of gestational age at clinical presentation including the presence or absence of the fetal phenotype [4]. 
The aim of this review is to provide an up-to-date narrative review on epidemiologic studies corroborating or refuting the hypothesis that maladaptation between the maternal immune system and the feto-placental "hemi-allograft" is involved in the causation of preeclampsia. For an overview of cellular and molecular aspects of the disease we would like to note some of the recently published reviews $[1,4]$.

Following some earlier more or less speculative papers by Mc Quarrie [8] and Veit [9] in the early 20th century, it was Medawar in 1953, who was the first to formally propose the concept of the feto-placental unit as an allograft [10]. Since then, it was assumed that implantation of the fetal placenta would be controlled by a maternal immune response mediated by $\mathrm{T}$ cells recognizing paternally derived allo-antigens expressed by the placenta. Ongoing research in the last decades has shown the major role of natural killer (NK) cells, the killer immunoglobulin-like receptors (KIR) and paternal human leukocyte antigen-C (HLA-C) [11], with more recently the discovery of a special subset of T-cells, the regular T (Treg) cells and their role as "master controller" while also providing a cellular basis for memory, and partner specificity. This special issue commemorates the enormous contributions of Gerard Chaouat, the "godfather of reproductive immunology". In the field of preeclampsia research, the recognition of the primipaternity paradigm and the protective role of prolonged sperm exposure were definitely some key factors triggering Gerard's interest in the study of this enigmatic syndrome.

\section{Primipaternity versus the birth interval hypothesis}

According to Leon Chesley, the "godfather" of preeclampsia research, genuine preeclampsia is a disease of first pregnancies. Leon Chesley was quite strict on this and clearly stated that serious preeclampsia researchers should really focus on the hypertensive syndrome occurring in first pregnancies, he also noted that a diagnosis of preeclampsia in a multiparous woman was mostly incorrect [12]. It was well recognised by obstetricians that a woman with a prior normal pregnancy is at a particularly low risk preeclampsia in her second pregnancy, even a previous abortion appeared to provide some protection in this respect $[12,13]$. But the key question is-is this protective effect of multiparity lost with a change of partner? As early as 1975, Need [14] found that in a patient's first pregnancy without preeclampsia, the response of maternal lymphocytes against lymphocytes of the first father was much less than against the 2nd father of the subsequent pregnancy which was complicated by severe preeclampsia. Following this, Feeney et al. [15] (1980) could only find 47 patients with preeclampsia (all severe) in a retrospective cohort of 34,000 multigravida deliveries with prior normal pregnancies. Out of these 47 multiparous patients with preeclampsia, 13 (28\%) patients had conceived in a new sexual relationship compared with only $4.3 \%$ in the large control group. Unfortunately, and this is the same for most studies reviewed in the current paper, no specifics are provided on the gestational age of these preeclamptic case. Interestingly, Feeney and Scott [16] also described that the incidence of preeclampsia in 125 primigravidas who had received massive blood-transfusions prior to pregnancy was significantly less than in a series of matched controls, and concluded that immunological factors may be involved in the aetiology of preeclampsia. After some conflicting small Nigerian studies $[17,18]$, Olayemi et al. [19] conducted a large prospective cohort study in 2 centers in Ibadan, a tertiary unit serving primarily a large city population and a regional referral hospital serving a predominantly rural population. All patients were nulliparous, while the focus of this study was on the effect of prior termination of pregnancies. In this prospective cohort of almost 2,000 patients, a prior same paternity termination of pregnancy was associated with a strong protective effect against developing preeclampsia [odds ratio (OR) 0.48; 95\% confidence interval (CI): 0.31-0.73], while prior different paternity terminations had no effect whatsoever. Data on preeclampsia subtype or pregnancy interval were not provided.

Robillard [20], as neonatologist working in a Guadeloupe Neonatal Intensive Care Unit (NICU) in the early 1990s was struck by the fact that many mothers of their preterm growth restricted infants born from preeclamptic pregnancies were multiparous. This appeared to directly contradict the then existing dogma of preeclampsia being a disease of first pregnancies. Robillard et al. [20] decided to explore the relationship between severe preeclampsia and changes in paternity patterns among Guadeloupe's multigravidas and found that 21 out of 34 (61.7\%) of the multiparous mothers with severe early-onset preeclampsia had a new 
partner compared with 4 out of $40(10 \%)$ among chronic hypertensive women and 10 out of $60(16.6 \%)$ in the control group $(P<0.0001)$. Because the patterns of changing paternity were significantly correlated preeclampsia in multipara but not with multiparous women with chronic hypertension and controls, the authors proposed that preeclampsia may be a problem of primipaternity rather than primigravidity. Importantly, all preeclamptic cases in this series had preterm preeclampsia [20].

Because Robillard's paper [20] did not provide evidence of documented significant proteinuria (in the 1990s still obligatory for the diagnosis of preeclampsia) in all preeclamptic patients, concerns were expressed regarding the validity of the diagnosis. Tubbergen et al. [21] decided to repeat Robillard's study using very strict diagnostic criteria. These Amsterdam researchers studied 333 multiparous patients with severe preterm preeclampsia and/or hemolysis, elevated liver enzymes and low platelet (HELLP) syndrome. The control group consisted of 182 multiparous women with uncomplicated pregnancies. The rate of pregnancies with new paternities was significantly higher $(P<0.0001)$ both for preeclamptic and HELLP patients in comparison with the controls with an OR of 8.6 (95\% CI: 3.1-23.5) and 10.9 (95\% CI: 3.7-32.3), respectively. Rather than using a case-control approach, Trupin et al. [22] studied a cohort of 5,068 nulliparas and 5,800 multiparas, 573 of whom had new partners. The preeclampsia incidence in nulliparas (3.2\%) and multiparas with changed paternity (3\%) was found to be similar, compared with the significantly lower preeclampsia incidence $(1.9 \%)$ in multiparas with no change in partners. Similar to many other large epidemiological studies no details were provided on the preeclampsia phenotype.

Skjaerven et al. [23] in 2003 were the first to formally challenge the "primipaternity" paradigm. Using the excellent Norwegian Birth Registry, they analysed the data on 551,478 women who had 2 or more singleton deliveries, and 209,423 women who had 3 or more singleton deliveries between 1967 and 1998. Preeclampsia occurred during $3.9 \%$ of first pregnancies, $1.7 \%$ of 2 nd pregnancies and $1.8 \%$ of 3 rd pregnancies in same paternity pregnancies. The investigators found that the risk in a 2 nd or $3 \mathrm{rd}$ pregnancy was directly related to the birth interval since the preceding delivery. An interbirth interval of $\geq 10$ years was associated with a preeclampsia risk similar to nulliparous women. For women with no previous preeclampsia, the risk of preeclampsia in 2nd pregnancy increased with increasing time interval. For intervals $\geq 15$ years, the OR was 2.11 (95\% CI: 1.75-2.53). The data on women with subsequent pregnancies in new relationships were more confusing; after controlling for the interbirth interval, changing paternity appeared to be associated with a reduced risk of preeclampsia (OR 0.80; 95\% CI: 0.72-0.90). But, the interaction between change in paternity and interbirth interval was only significant in women with no previous preeclampsia. Mysteriously, for women with previous preeclampsia, the risk tended to decrease with increasing birth interval. Skjaerven et al. [23] concluded that the primipaternity concept was wrong and stated that the increase in preeclampsia risk ascribed to a new father by others was due to insufficient control for interbirth interval. Similar to most other large epidemiologic studies, no data were provided on the preeclampsia phenotype. Using a large Danish cohort, Basso et al. [24] also found that prolonged birth interval is a risk factor for preeclampsia. Interestingly, these Danish authors did note that changing paternity in patients with prior preeclampsia appeared to be protective after stratifying for the interpregnancy interval.

Also, Mostello et al. [25] conducted a study focussed on identifying risk factors for preeclampsia in second pregnancies using data from the 1989-1997 birth registry in Missouri; 2,332 cases with preeclampsia in the 2nd pregnancy, and 2,370 control cases were analysed. A history of prior preeclampsia conferred the highest risk, longer birth intervals were also a significant risk factor, but in contrast to the Norwegian study, having the 2nd baby with the same father paternity was found to be protective.

Conde-Agudelo and Belizan [26] analysed data on 456,889 Latin-American parous women delivering singleton infants. Birth intervals of $>59$ months were observed in $19.5 \%$ of these multiparous women; these long birth intervals were associated with significantly increased risks of preeclampsia $(1.83 ; 1.72-1.94)$ and eclampsia $(1.80 ; 1.38-2.32)$.

In our 2003 review [27], we explained our concerns and explored alternative explanations for the Norwegian findings [23] and argued that the "primipaternity" concept is still standing. Besides the intrinsic inaccuracy of this type of birth registry, the authors also claimed that the diagnosis of preeclampsia in their 
study included the presence of $0.3 \mathrm{~g}$ of protein per $24 \mathrm{~h}$. Clearly, Skjaerven et al. [23], being epidemiologists, failed to recognise that most Western countries only started to use 24-h urine, or protein/creatinine ratios in the 1980s. The diagnosis is therefore questionable in at least $60 \%$ of their "preeclamptic" multiparous patients, since as Chesley stressed [12] the diagnosis of mild preeclampsia is usually erroneous in multiparous women, in particular in the absence of proper quantification of the degree of proteinuria. While Skjaerven et al. [23] argued against the aforementioned studies of Robillard et al. [20] and Tubbergen et al. [21] because of their relatively small sample size, he failed to discuss the findings of a 2000 study published by Li and Wi [28]. These authors [28] reported a cohort study based on 140,147 women with two consecutive births during 1989-1991 identified through linking of annual Californian birth certificate data. Among women without preeclampsia/eclampsia in the 1st pregnancy, changing partners resulted in a $30 \%$ increase in the risk of preeclampsia/eclampsia in the subsequent pregnancy compared with those who did not change partners (95\% CI: 1.1-1.6). On the other hand, among women with preeclampsia in the 1st birth, changing partners resulted in a 30\% reduction in the risk of preeclampsia in the subsequent pregnancy $(95 \% \mathrm{CI}: 0.4-$ 1.2). Interbirth interval was very unlikely to be a confounder in the Li and Wi study [28] since the authors restricted their population to births that were between 1-3 years apart. Interestingly, other Norwegian authors, in an earlier report [29] on the same Norwegian cohort used by Skjaerven et al. [23], also found that the risk to develop preeclampsia for mothers who had had preeclampsia in the 1 st pregnancy was $13.1 \%$ if she had her 2nd pregnancy with the same partner, the risk dropping to $11.8 \%$ if she changed the partner. These findings appear to be biologically inconsistent with the birth interval hypothesis. If anything, one would expect a longer birth interval after experiencing a traumatic life event such as a pregnancy complicated by preeclampsia in combination with the time required to find a new partner. So, this aspect of the Norwegian database analysis and the aforementioned Californian data [28] appear to disagree with the birth interval hypothesis. Importantly, another publication [30] preceding the 2002 landmark New England Journal of Medicine publication [23] on exactly the same population cohort, but with different authors, specifically emphasized that prolonged interbirth interval is not a risk factor in women with previous preeclampsia with same or new paternity pregnancies. Again, these findings contradict the birth interval hypothesis. It is hard to conceive of any risk factor associated with prolonged interbirth interval [environmental, infection, stress, body mass index (BMI), etc.] increasing the risk in women with previously normal pregnancies while decreasing the risk in women with previous preeclamptic pregnancies.

But, although there are certainly still issues unresolved with the "birth interval" hypothesis, what could be the likely explanations for the findings of these large epidemiological studies?

(1) These birth registries do not register miscarriages. The occurrence of one or more miscarriages, a very common event for many women in Western societies, may explain a significant proportion of the couples apparently "opting" for a prolonged birth interval. Women with recurrent miscarriages are known to have a higher incidence of adverse pregnancy outcome in subsequent ongoing pregnancies [31].

(2) Prolonged interbirth interval could also reflect secondary subfertility, with polycystic ovary syndrome (PCOS) and major obesity perse as common causes. Both PCOS and increased BMI have clear associations with preeclampsia in subsequent pregnancies $[6,32]$, while even unexplained infertility has been identified as a risk factor for preeclampsia [33]. The aforementioned group of Danish researchers [25] who had studied the effect of prolonged birth interval as a risk factor, also came to this conclusion after focussing on subfecundity as a risk factor for preeclampsia in the Danish population [34]. Among women with no known hypertension, the authors found a higher risk of preeclampsia in those with longer times to pregnancy, after adjustment for maternal age, pre-pregnancy body mass index, and smoking. Finally, the effect of miscarriages and fertility problems were also specifically addressed in another Norwegian study by Trogstad et al. [35] using the data from the 1999-2005 MoBa cohort (Norwegian Mother and Child Cohort Study). An increased risk of preeclampsia, although not statistically significant, was found for women with recurrent miscarriages (adjusted OR 1.51; 95\% CI: 0.80-2.83). Women who had ever been treated for infertility also had increased risk (adjusted OR 1.29; 95\% CI: 1.05-1.60). When these two risk factors were combined, the adjusted OR for preeclampsia was 2.40 (95\% CI: 1.11-5.18). 
In the discussion section of her excellent study on the effect of prior abortions, Saftlas et al. [36] also emphasized that the Scandinavian investigators [23] ignored the effect of history of abortions, particularly induced. Saftlas et al. [36] showed that women with a history of abortion who conceived again with the same partner had nearly half the risk of preeclampsia (OR 0.54; 95\% CI: 0.31-0.97). In contrast, women with an abortion history who conceived with a new partner had the same risk of preeclampsia as women without a history of abortion. These data were from the 1992-1995 Calcium for Prevention of Preeclampsia (CPEP) trial, which enrolled only nulliparous women (median age, 19.7 years), the average interpregnancy interval approximated 1 year, given that the average age of primigravidas was 1 year less than that for women who had one previous pregnancy. Saftlas et al. [36] stressed that confounding by inter-pregnancy interval is also unlikely because the ORs for women above or below the median age were virtually identical, despite the fact that the interpregnancy interval was, of necessity, shorter for women below than above the median age. In addition, the average age of women who changed partners was only about a month more than that of women who remained with the same partner. Saftlas et al. [36] stressed that failure to account for terminations of pregnancies conceived between registered births would result in erroneously long interbirth intervals attributed to women who change partners.

Also, Trogstad et al. [37] looked at the effect of prior abortions in nulliparous pregnant women using data from the aforementioned Norwegian MoBa cohort. Abortions were defined as all pregnancies ending prior to 22 week's gestation. When compared with primigravida women, one previous induced abortion tended to reduce the risk of preeclampsia for women with same paternity pregnancies but not for women with new paternity pregnancies, however the confidence intervals were wide due to small numbers. For both same paternity and new paternity pregnancies two or more previous induced abortions tended to reduce the risk of preeclampsia. The risk appeared to be lower in same paternity as compared with new paternity pregnancies. For women with two or more previous spontaneous abortions, a change in paternity tended to lower the estimated risk of preeclampsia as compared with spontaneous abortions with unchanged paternity, and compared with primigravid women, but again the confidence intervals were wide. The authors concluded that normal pregnancies interrupted in early pregnancies may induce immunological changes that reduce the risk of preeclampsia in a subsequent pregnancy.

Hercus et al. [38] studied a retrospective cohort of 2,003 multiparous women with detailed access to medical records and demonstrated that both primipaternity and prolonged birth and/or pregnancy interval act as independent risk factors for preeclampsia in multigravida with a similar effect size. In addition to the aforementioned indirect ways, there may be additional direct biologicals effect by which prolonged birth intervals may increase the risk for preeclampsia in multiparous women. Loss of the "pantyhose phenomenon" has been suggested, with the loss of the modification, or "stretching," of the proximal spiral artery created by the prior pregnancy with a greater time interval. Khong et al. [39] demonstrated that structural changes of the proximal spiral arteries needed for the pregnancy, do not completely resolve following parturition, and that the duplication and fragmentation of the internal elastic lamina and the proportion of non-muscular tissue increased with increasing parity. It is currently unknown if these changes regress with prolonged birth intervals. Alternatively, fading T-cell memory could play a role. Pregnancy-induced memory in T cells and uterine NK cells [1] likely contributes to the protective effect of prior pregnancy. Saito and his collaborators [40] proposed loss of memory T cell "memory" over more prolonged interval as an attractive alternative explanation.

In summary: both primipaternity and prolonged interbirth intervals are risk factors for preeclampsia. Prolonged birth intervals are probably more related to the late-onset preeclampsia (since these are by far the most common subtype in the large epidemiologic studies), while primipaternity appear to be more related to early-onset preeclampsia, i.e., the phenotype of preeclampsia associated with superficial CTB invasion in the spiral arteries. 


\section{Sperm exposure}

The protective effective of repeated sperm exposure was first described by Marti and Herrmann [41].

These German researchers, in a painstaking study, counted the number of "sperm exposures" in women with uncomplicated pregnancies compared with women developing preeclampsia; 28 primigravida with preeclampsia, and 55 with uncomplicated pregnancies were included. The preeclamptic patients had an average of 59.5 episodes of vaginal intercourse while the non-preeclamptic control group had 191.6 exposures. Oral contraceptives (allowing sperm exposure) and the total period of oral contraceptive use was significantly lower in the preeclamptic women. The authors stated that their findings might provide an explanation for the high incidence of preeclampsia in teenage pregnant girls. However, in the 1970s, preeclampsia in Germany was still defined by a gestosis index score [42]. The gestosis index [42] combined systolic and diastolic blood pressure, proteinuria and edema to classify patients as having mild, moderate or severe edema-proteinuria-hypertension (EPH) gestosis. As such, a woman with a bit more pronounced physiologic edema would have been diagnosed as having mild EPH gestosis. The issue of sperm exposure protecting against preeclampsia was reassessed by Klonoff et al. [43] comparing the contraceptive and reproductive histories of 110 primiparous women with preeclampsia (using more contemporary definitions) to 115 pregnant women without preeclampsia (controls matched to cases by age, race, and distance from the hospital). The use of barrier contraceptives (and withdrawal) was associated with an increased risk of preeclampsia (OR 2.37; 95\% CI: 1.01-5.58); according to Klonoff et al. [43], these contraceptive methods could "explain" $60 \%$ of preeclamptic cases. In another case control study, Einarsson et al. [44] compared 113 preeclamptic cases with 226 controls (matched by age and parity); women with a short period of cohabitation ( $<4$ months) using barrier methods for contraception had a substantially elevated risk (OR 17.1; $P=0.004$ ) for development of preeclampsia compared with women with more than 12 months of regular sexual intercourse prior to conceiving.

Robillard et al. [45] completed the first large retrospective cohort study on the relationship between sperm exposure and preeclampsia; 1,011 consecutive women of mixed parity delivering in one obstetric unit on the Caribbean Island of Guadeloupe were postnatally interviewed about paternity and duration of sexual cohabitation before conception. The incidence of pregnancy-induced hypertensive disorders was $11.9 \%$ among primigravidae, $4.7 \%$ among samepaternity multigravidas, and $24.0 \%$ among newpaternity multigravidas. The length of sexual cohabitation before conception was inversely related to the incidence of a pregnancy-induced hypertensive disorder $(P<0.0001)$ in both primigravida and multigravida. Compared with women with regular sexual intercourse $\geq 12$ months as a reference, the adjusted OR for developing a pregnancy-induced hypertensive disorder, when the period of regular sexual intercourse was 0-4 months was 11.6 (95\% CI: 6.4-20.9), for a period of 5-8 months 5.9 (95\% CI: 2.9-12.5), and for a period of 9-12 months 4.2 (95\% CI: 1.7-10.4). Reflective of the typical reproductive pattern in Guadeloupe, Robillard et al. [45] demonstrated that those multigravidas with a pregnancy-induced hypertensive disorder had a new partner in $66.7 \%$ of cases compared with $24.1 \%$ in normotensive multigravidas $(P=0.0001)$. The very high incidence $(24.0 \%)$ of pregnancy-induced hypertension among newpaternity multigravidas was shown to be related to a remarkably short period of sperm exposure preceding conception. A problem with this study was that while 21 patients developed proteinuric pregnancy-induced hypertension (preeclampsia and/or eclampsia), the other 81 patients developed gestational hypertension. In the analysis, these patients were lumped together as pregnancy-induced hypertensive disorders.

In a well-designed study, Ness et al. [46], asked 2,211 women about preconception contraception and timing of first sexual intercourse with the father of the pregnancy. OR's comparing cases with preeclampsia to the rest of the cohort were adjusted for age, smoking, parity, and BMI. In this Pittsburgh population, women using barrier contraception prior to conception were no more likely than women not using barrier contraception to develop preeclampsia (adjusted OR 1.0; 95\% CI: 0.6-1.6). In unadjusted analyses, a prolonged time to conception was associated with preeclampsia (OR 1.9), however, after adjustment, the association was less prominent (OR 1.6) and after stratification by contraception method, the link between time to conception and preeclampsia was eliminated. Obviously, the Pittsburgh researchers concluded that 
their data do not support the protective effect of prolonged sexual relationship prior to conceiving. It should be noted that some of the data in the Ness et al. [46] study appear to be inconsistent with known biological facts. The most important being a 1st month conception rate of 40-55\%; this type of data makes it at least a bit difficult to accept the validity of the raw data used for their analysis [47].

As is true in most epidemiologic studies, the Ness et al. [46] study provides no data on presence or absence of IUGR, or early- versus late onset preeclampsia. In the Pittsburgh population with a high rate of obesity, term preeclampsia was almost certainly the most common phenotype. As mentioned earlier, the fundamental question addressing the immune maladaptation is best addressed by focussing on pregnancy complications associated with superficial CTB invasion in the spiral arteries. It was the Screening for Pregnancy Endpoints (SCOPE) group [48] using the data from their large prospective cohort study of 5,591 healthy nulliparous women, demonstrating that a short duration of sexual relationship is an independent risk factor for pregnancies being complicated by small for gestational age (SGA) in the presence of abnormal uterine artery Doppler indices at $20 \pm 1$ weeks'. This study also showed that abnormal uterine artery Doppler resistance indices at $20 \pm 1$ weeks are more common among women who have a short duration of sexual relationship. Overall preeclampsia had a non-significant trend with short sexual relationship, again showing that most cases of preeclampsia seen nowadays in Western countries have late-onset preeclampsia, i.e., the type of preeclampsia not related to superficial CTB invasion. Indeed, in this large prospective cohort, the group of preeclamptic women who also had abnormal uterine artery Doppler (52 out of total of 277 preeclamptic patients) also had a shorter duration of sexual relationship but this large prospective cohort had insufficient power.

Saftlas et al. [49] looked at cumulative sperm exposure (length of relationship $\times$ average sexual intercourse frequency). In a study of 340 nulliparous women ( 258 preeclampsia cases, 182 controls), women in the highest 10th centile of exposure to the partner's sperm and seminal fluid had $70 \%$ reduced odds of preeclampsia relative to women in the lowest 25th percentile. Finally, Zhu et al. [50] recently published a Chinese study: 502 single birth primigravid women were divided into two groups according to the duration of sperm exposure with the biological father, short duration of sperm exposure ( $\leq 3$ months) and long duration of sperm exposure ( $\geq 12$ months; $n=122$ ). Patients with short duration of sperm exposure had a nonsignificantly higher risk of any type of pregnancy-induced hypertensive disorders compared with patients with sperm exposure longer than 12 months (OR 2.34; 95\% CI: 0.97-5.65), while the risk of preeclampsia was markedly increased in short duration group (OR 7.44; 95\% CI: 0.99-55.75).

So, what is the biological explanation underlying the protective effect of regular sperm exposure? Reproductive immunologists, led by Sarah Roberson in Adelaide [1] demonstrated that regular sperm exposure triggers a partner-specific mucosal tolerance. Seminal fluid transforming growth factor $\beta$ (TGF $\beta$ ) and the partner specific antigen is proposed to inhibit the induction of type 1 immune responses against the semi-allogenic conceptus that are thought to be associated with impaired placentation and spiral artery remodelling which are features of early-onset preeclampsia and intrauterine growth restriction [2, 4]. The exact way by which the woman is exposed to the paternal HLA message was initially uncertain.

Koelman et al. [51] demonstrated the presence of soluble class I human leukocyte antigen (HLA) molecules in seminal plasma representing a potential straightforward way of endometrial exposition. Interestingly, soluble HLA molecules have been demonstrated to induce apoptosis in human cytotoxic $\mathrm{T}$ cells [52]; induction of apoptosis may be a mechanism in inducing specific tolerance against the partners HLA cell membrane molecules. In contrast, findings in studies on pregnancies following intra-uterine insemination [53], concluded that the protective factor appeared mediated by the actual spermatozoa and not the seminal fluid. Wang et al. [54] used the very elegant model of intra-cytoplasmatic sperm injection (ICSI) pregnancies to confirm the protective effect of previous sperm exposure and to analyse whether or not this protection is conveyed by sperm cells or seminal plasma. Pregnancy outcome in a cohort of pregnancies achieved after (ICSI), including couples with male partners with azoospermia (surgically obtained sperm cells) were evaluated. The azoospermia group provides an ideal "model" to test the protective partner-specific immune-tolerance conveyed by sperm cells independent of what provided by seminal fluid. In these couples 
the exposure of the female genital tract to sperm cells during intercourse is absent, while the exposure to seminal fluid is not affected. Altogether, 1,621 deliveries conceived after standard in vitro fertilization (IVF), ICSI using sperm cells obtained by masturbation, and ICSI using surgically obtained sperm cells were analysed; $195(12.0 \%)$ had gestational hypertension, and 67 of them (4.1\%) had preeclampsia. The risk of gestational hypertension (22\% versus $11 \%$ ) was doubled, while the risk of preeclampsia was tripled $(11 \%$ versus $4 \%)$ in ICSI using surgically obtained sperm compared with standard IVF and ICSI using sperm obtained by masturbation. This study clearly confirms that previous exposure to the actual sperm cells must convey a major part of the protection, since women in the ICSI group using sperm cells obtained by transcutaneous surgical methods clearly never had any previous contact with their partner's sperm cellsand it was only this subset of patients with longstanding infertility that had the increased incidence of both preeclampsia and gestational hypertension.

The Adelaide group of researchers led by Sarah Robertson demonstrated that [55], both in animal studies but also in smaller human studies using post intercourse cervical biopsies, deposition of semen in the female genital tract provokes a cascade of cellular and molecular events resembling a classic inflammatory response. Seminal TGF1 appears to be the critical factor. Seminal vesicle-derived TGF1 is secreted predominantly in a latent form. Seminal plasmin and uterine factors transform the latent form into bioactive TGF1 [55]. Intra-uterine insemination of TGF1 in-vivo results in an increase in granulocyte-monocyte colony stimulating factor (GM-CSF) production that is sufficient to initiate an endometrial leucocytosis quite similar to effects of mating [56]. The introduction of TGF1 into the uterus in combination with paternal ejaculate antigens favours the growth and survival of the semi-allogenic fetus, as evidenced by a significant increase in fetal and placental weight in animal studies, in two ways. Firstly, by initiating a post-mating inflammatory reaction, TGF1 increases the ability to sample and process paternal antigens contained within the ejaculate. Another important role of TGF1 and the subsequent post-coital inflammatory response is the initiation of a strong type 2 immune deviation. The processing of an antigen by antigen-presenting-cells in an environment containing TGF1 is likely to initiate a T helper 2 (Th2) phenotype within these responding T-cells [56]. By initiating a type 2 immune response towards paternal ejaculate antigens, seminal TGF1 may inhibit the induction of type 1 responses against the semi-allogenic conceptus that are thought to be associated with poor placental and fetal development. Decidual macrophages, present in an immune-suppressive phenotype from the moment of implantation, may inhibit NK cell lytic activity through their release of molecules such as TGF, interleukin-10 (IL-10) and prostaglandin-E2 (PGE2). Under the influence of the local cytokine environment, antigen-presenting-cells such as macrophages and dendritic cells may take up, process and present ejaculate antigens (sperm, somatic cells, and soluble antigens) to T cells in the draining lymph nodes loss [55]. Sperm also assists in promoting female immune tolerance by eliciting uterine cytokine expression through a Toll-like receptor 4 (TLR4)-dependent signalling [1].

Clearly, not all pregnancies after short sexual relationships are at risk of preeclampsia. It is currently not known whether or not these couples represent examples of ideal immune matching between a specific maternal NK cell KIR receptor repertoire and placental (paternal) HLA-C expression. It should also be noted that the placenta itself also plays an important role in immune tolerance in healthy pregnancies. Besides progesterone many placental proteins particularly several galectins play a pivotal role. Placental protein 13 (PP13), discovered by Hans Bohn in the early 1970's, is one of these galectins [57]. Some galectins are solely expressed in the placenta, where they may confer additional immunoregulatory functions to enable deep placentation. PP13 is released by the STB and has the ability to induce apoptosis of activated T cells, and to divert and kill T cells as well as macrophages in the maternal decidua. Lower levels of PP13 in early first trimester maternal sera are associated with elevated risk of preeclampsia [57].

\section{Donor insemination, oocyte donation and embryo donation}

Artificial donor insemination represents an "iatrogenic replica" of very short sperm exposure. Several investigators have reported on the substantially increased rate of preeclampsia after donor insemination [58]. Need et al. [59] were the first, reporting on the course of pregnancy of 584 pregnancies achieved in artificial 
donor insemination (ADI) programmes throughout Australia. The overall incidence of preeclampsia was high (9.3\%) compared with the expected incidence of $0.5-5 \%$. The incidence was increased in both multigravid and primigravid women. The expected preeclampsia incidence in Australian primigravid women was 5\%, while it was $10.1 \%$ in primigravid women pregnant following donor insemination. In the multigravid women, the differences were even more striking; the expected preeclampsia incidence was $0.9 \%$ in multigravid patients, while it was $7.8 \%$ in the multigravid patients following donor insemination. Thus, the expected protective effect of a previous pregnancy was not seen, with a 47-fold increase in preeclampsia (observed versus expected) in donor-insemination pregnancies after a previous full-term pregnancy and a 15 -fold increase after a pregnancy of short duration. Grefenstette et al. [60] published a national French study on 470 pregnancies following ADI using frozen sperm, and also reported a significantly increased incidence of pregnancy-induced hypertensive disorders compared with a control group.

Serhal and Craft [61] in 1987 were the first to report on the effect of donor oocytes in their first 10 pregnant patients; 5 out of these 10 previously normotensive women developed preeclampsia. In a follow-up study in 1989, on 61 pregnancies achieved after oocyte donation, the same authors found a preeclampsia rate of 38\% [62]. Salha et al. [63] reported on 72 women who had conceived with the use of different types of donated gametes compared with 72 women (age- and parity-matched) who became pregnant with their own gametes, either spontaneously, or following intrauterine insemination with their partner's spermatozoa. The incidence of pregnancy-induced hypertension in the donated gametes study group was $12.5 \%(9 / 72)$ compared with $2.8 \%(2 / 72)$ in the control group. In addition, preeclampsia was diagnosed in $18.1 \%(13 / 72)$ of the donated gametes study group compared to $1.4 \%(1 / 72)$ in the age- and parity-matched controls. In the 12 women who conceived with donated embryos 4 (33.3\%) developed hypertension compared with none of their age and parity matched controls. There are currently multiple studies confirming the increased risk for preeclampsia in oocyte donation pregnancies. Blazquez et al. [64] compared double gamete donation (embryo donation) with oocyte donation in a cohort study of 433 patients. These authors demonstrated that double gamete donation pregnancies are a particularly higher risk of early-onset preeclampsia (OR 3.02; 95\% CI: 1.11-8.2), while the authors did not find a difference for term preeclampsia.

A recent systematic analysis and meta-analysis was recently published by Moreno et al [65]. Twenty-three studies were included. IVF-oocyte donation was found to be associated with a higher risk of hypertensive disorders in pregnancy (OR 2.63; 2.17-3.18), preeclampsia (OR 2.64; 2.29-3.04), severe preeclampsia (OR 3.22 ; 2.30-4.49), gestational hypertension (OR 2.16; 1.79-2.62), preterm birth (OR 1.57; 1.33-1.86), early preterm birth (OR 1.80; 1.51-2.15), low birth weight (OR 1.25; 1.20-1.30), very low birth weight (OR 1.37; 1.22-1.54), and gestational diabetes (OR 1.27; 1.03-1.56). There was no significant difference in the risk of preterm birth or low birth weight when adjusted for preeclampsia.

In summary, in line with the immune maladaptation hypothesis, pregnancies on the basis of donated gametes, and even more so donor embryo pregnancies are at a clearly increased risk of preeclampsia. With the increasing importance of this type of reproductive techniques in the foreseeable future, clinical obstetricians need to adapt their antenatal care in these higher-risk pregnancies.

\section{Conclusion}

Nowadays, term preeclampsia is by far the most commonly encountered phenotype of preeclampsia; women at time of their first babies are 10-15 years older, and mostly had lengthy sexual relationships before conceiving. Couple-specific immune maladaptation probably plays no or only a very minimal role in this preeclampsia phenotype. As mentioned, the main drivers appear to be increased maternal BMI, increased gestational weight gain and other clinical characteristics making up the metabolic syndrome, and maternal age [6, 7]. The fact that this is by far the most common phenotype led some researchers to conclude that preeclampsia is caused by a "poor" pre-pregnancy cardiovascular/metabolic status-and for the term phenotype this is probably largely true. The studies reviewed in this paper strongly indicate that immune maladaptation is involved in the aetiology of preeclampsia, or to be more precise early-onset preeclampsia being associated with superficial CTB invasion in the spiral arteries and IUGR. The prematurely stressed STB will release a 
host of pro-inflammatory mediators (type I cytokines) and importantly the STB release of PLGF decreases while the release of sFlt-1 and soluble endoglin (sEng) increase. These factors-in combination-cause the final characteristic systemic endothelial cell dysfunction, particularly in specialized endothelial cells like the podocytes, the Kupfer cells in the liver sinusoids and the choroid plexus. The conclusions derived from the studies we reviewed in this paper may have practical consequences for practising physicians, even while the exact aetiology and pathogenesis of the different preeclampsia phenotypes still needs to be resolved:

(1) According to the primipaternity concept, a multiparous woman with a new partner should be approached as being a primigravid woman.

(2) Artificial donor insemination, oocyte donation and especially embryo donation are associated with an increased risk of developing pregnancy-induced hypertensive disorders.

(3) A more or less prolonged period of sperm exposure provides a partial protection against pregnancy-induced hypertensive disorders. Nowadays, women with changing partners are strongly advised to use condoms in order to prevent sexually transmitted diseases. However, a certain period of sperm exposure within a stable relation, when pregnancy is aimed for, is associated with a partial protection against preeclampsia

The observation of an inverse relationship between the duration of sexual cohabitation and the incidence of preeclampsia suggests that long-term sperm exposure may be important for human implantation success $[47,66]$. The human female typically has "concealed ovulation" and is one of the few mammals exposed to her partner's semen on multiple occasions prior to conceiving. From an evolutionary perspective, it can be argued that induction of paternal antigen tolerance through repeated sperm exposure may have reproductive advantages, perhaps by promoting implantation and survival of embryos conceived in long-term relationships where possibly the male parent may be more committed to the well-being of the resultant child. Preeclampsia is a uniquely human disease, and throughout evolution the relatively high incidence of preeclampsia must have represented a significant reproductive disadvantage in humans compared with other mammals. In developing countries nowadays and until the 1950s in developed countries, eclampsia accounted for at least $1 \%$ of human births $[47,66]$. Not surprisingly evolutionary biologists proposed that in line with its high incidence, preeclampsia might "hide an adaptive advantage" somewhere. This may be true for the increase in blood pressure per se, since the increased maternal systemic blood pressure will increase perfusion pressure [67] in line with Haig's pregnancy as a genetic conflict paradigm [68]. According to Robillard et al. [47, 66], another major difference between the human embryo and their mammal counterparts is the size of the fetal brain requiring about $60 \%$ of total fetal nutritional needs during the extraordinary phase of brain development in the 2nd and 3rd trimester of pregnancy, and he hypothesized that the large size of the human fetal brain requires the deep endovascular trophoblast invasion. According to this hypothesis, such a deep endovascular trophoblast invasion can only occur on the basis of major immunogenetic compromises in terms of maternal-paternal tissue tolerance. Preeclampsia was the price humankind had to pay when immunogenetic compromises were not achieved in some couples.

While this is certainly an attractive hypothesis, alternative viewpoints explaining the risks of changing partners, and new partner short sperm exposure were proposed by Kell and Kenny [69]. In their excellent review, these authors discussed the data suggesting that a dormant microbial played a role in the development of preeclampsia. A new sexual partner would obviously expose the women to a new "partner-specific" microbiome. Indeed, many studies reviewed in the Kell and Kenny paper [68] have identified a much greater prevalence of infectious agents in the blood and/or urine in preeclamptic patients compared with controls. This hypothesis is not directly contradicting the role of partner specific immune maladaptation. Recent, evidence in mice that seminal fluid regulates Treg cells (priming), including proliferation and reinforcing a suppressive phenotype through epigenetic modulation. Priming may be dysregulated due to seminal fluid composition as may occur in infections. As such a disturbed microbiome (microbiome dysbiosis) could still operate via triggering a disturbed or lacking partner-specific mucosal tolerance [1]. 


\section{Abbreviations}

BMI: body mass index

CI: confidence interval

CTB: cytotrophoblast

HLA: human leukocyte antigen

ICSI: intra-cytoplasmatic sperm injection

IUGR: intra-uterine growth restriction

IVF: in vitro fertilization

OR: odds ratio

PLGF: placental growth factor

PP13: placental protein 13

sFlt-1: soluble fms-like tyrosine kinase-1

STB: syncytiotrophoblast

TGF $\beta$ : transforming growth factor $\beta$

\section{Declarations}

\section{Author contributions}

GAD: primary author, literature search, writer; PYR: second author, writing, criticizing and proofreading.

\section{Conflicts of interest}

The authors declare that they have no conflicts of interest.

\section{Ethical approval}

Not applicable.

\section{Consent to participate}

Not applicable.

\section{Consent to publication}

Not applicable.

Availability of data and materials

Not applicable.

\section{Funding}

Not applicable.

\section{Copyright}

(C) The Author(s) 2021.

\section{References}

1. Robertson SA, Green ES, Care AS, Moldenhauer LM, Prins JR, Hull ML, et al. Therapeutic potential of regulatory T cells in preeclampsia-opportunities and challenges. Front Immunol. 2019;10:478.

2. Redman CWG. Pre-eclampsia: a complex and variable disease. Pregnancy Hypertens. 2014;4:241-2.

3. Redman CWG, Staff AC, Roberts JM. Syncytiotrophoblast stress in preeclampsia: the convergence for multiple pathways. Am J Obstet Gynecol. 2020;S0002-9378:31115-7. 
4. Burton GJ, Redman CWG, Roberts JM, Moffett A. Pre-eclampsia: pathophysiology and clinical implications. BMJ. 2019;366:12381.

5. Grieger JA, Greskowiak LE, Wilson RL, Bianco-Motto T, Leemaqz SY, Jankovic-Karasoulos T, et al. Metabolic syndrome in pregnancy and risk for adverse pregnancy outcomes: a prospective cohort of nulliparous women. PLoS Med. 2018;15:e1002710.

6. Robillard PY, Dekker G, Sciosia M, Bonsante F, Iacobelli S, Boukerrou M, et al. Increased BMI has a linear association with late-onset preeclampsia: a population-based study. PloS One. 2019;14:e223888.

7. Robillard PY, Dekker G, Boukerrou M, Boumahni B, Hulsey T, Scioscia M. Gestational weight gain and rate of late-onset preeclampsia: a retrospective analysis on 57000 singleton pregnancies in Reunion Island. BMJ Open. 2020;10:e036549.

8. Mc Quarrie I. Isoagglutination in the new born infants and their mother: a possible relationship between interagglutination and the toxemias of pregnancy. Bull Johns Hopkins Hosp. 1923;34:51-4.

9. Veit J. über albuminurie in der Schwangerschaft. Ein Beitrag zur Physiologie der Schwangerschaft. Berliner Klin Wchschr. 1902;3:513-6. German.

10. Medawar PB. Some immunological and endocrinological problems raised by the evolution of viviparity in vertebrates. In: Symposium of the Society for Experimental Biology. 1953. pp. 320-38.

11. Hiby SE, Walker JJ, O'shaughnessy KM, Redman CWG, Carrington M, Moffett A. Combinations of maternal KIR and fetal HLA-C genes influence the risk of preeclampsia and reproductive success. J Exp Med. 2004;200:957-65.

12. Chesley L. Epidemiology of preeclampsia-eclampsia. In: Hypertensive disorders in pregnancy. Appleton Century Crofts. 1978. pp. 35-55.

13. Strickland DM, Guzick DS, Cox K, Gant NF, Rosenfeld CR. The relationship between abortion in the first pregnancy and development of pregnancy induced hypertension in the subsequent pregnancy. Am J Obstet Gynecol. 1986;154:146-8.

14. Need JA. Pre-eclampsia in pregnancies by different fathers. Br Med J. 1975;1:548-9.

15. Feeney JG, Scott JS. Pre-eclampsia and changed paternity. Eur J Obstet Gynecol Reprod Biol. 1980;11:35-8.

16. Feeney JG, Tovey LA, Scott JS. Influence of previous blood-transfusions on incidence of preeclampsia. Lancet. 1977;1:874-5.

17. Ikedife D. Eclampsia in multiparae. Br Med J. 1980;280:985-6.

18. Chigbu B, Okezie OA, Ogudu BU. Women in Southern Nigeria with change in paternity do not have increased incidence of pre-eclampsia. J Obstet Gynaecol. 2009;29:94-7.

19. Olayemi O, Strobino D, Adedapo K, Aimakhu C, Odukogbe AT, Salako B. Influence of previous abortions and new paternity on the risk of hypertension in nulliparous parturients in Ibadan: a cohort study. J Obstet Gynaecol Res. 2010;36:965-9.

20. Robillard PY, Hulsey TC, Alexander GR, Keenan A, de Caunes F, Papiernik E. Paternity patterns and risk of preeclampsia in the last pregnancy in multiparae. J Reprod Immunol. 1993;24:1-12.

21. Tubbergen P, Lachmeijer AM, Althuisius SM, Vlak ME, van Geijn HP, Dekker GA. Change in paternity: a risk factor for preeclampsia in multiparous women? J Reprod Immunol. 1999;45:81-8.

22. Trupin LS, Simon LP, Eskenazi B. Change in paternity: a risk factor for preeclampsia in multiparas. Epidemiology. 1996;7:24-4.

23. Skjaerven R, Wilcox AJ, Lie RT. The interval between pregnancies and the risk of preeclampsia. New Engl J Med. 2002;346:33-8.

24. Basso O, Christensen K, Olsen J. Higher risk of pre-eclampsia after change of partner. An effect of longer interpregnancy intervals. Epidemiology. 2001;12:624-9. 
25. Mostello D, Catlin TK, Roman L, Jr. Holcomb WL, Leet T. Preeclampsia in the parous woman: who is at risk. Am J Obstet Gynecol. 2002;187:425-9.

26. Conde-Agudelo A, Belizan JM. Maternal morbidity and mortality associated with interpregnancy interval: cross sectional study. Br Med J. 2000;321:1255-9.

27. Dekker G, Robillard PY. The birth interval hypothesis—does it really indicate the end of the primipaternity hypothesis. J Reprod Immunol. 2003;59:245-51.

28. Li DK, Wi S. Changing paternity and the risk of preeclampsia/eclampsia in the subsequent pregnancy. Am J Epidemiol. 2000;151:57-62.

29. Lie RT, Rasmussen S, Brunborg H, Gjessing HK, Lie-Nielsen E, Irgens LM. Fetal and maternal contributions to risk of pre-eclampsia: a population based study. BMJ. 1998;316:1343-7.

30. Trogstad LI, Eskild A, Magnus P, Samuelsen SO, Nesheim BI. Changing paternity and time since last pregnancy; the impact on pre-eclampsia risk. A study of 547238 women with and without previous pre-eclampsia. Int J Epidemiol. 2001;30:1317-22.

31. Jivraj S, Anstie B, Cheong YC, Fairlie FM, Laird SM, Li TC. Obstetric and neonatal outcome in women with a history of recurrent miscarriage: a cohort study. Hum Reprod. 2001;16:102-6.

32. Vries de MJ, Dekker GA, Schoemaker J. Higher risk of preeclampsia in the polycystic ovary syndrome. A case control study. Eur J Obstet Gynecol Reprod Biol. 1998;76:91-5.

33. Pandian Z, Bhattacharya S, Templeton A. Review of unexplained infertility and obstetric outcome: a 10-year review. Hum Reprod. 2001;16:2593-7.

34. Basso O, Weinberg CR, Baird DD, Wilcox AJ, Olsen J; Danish National Birth Cohort. Subfecundity as a correlate of preeclampsia: a study within the Danish National Birth Cohort. Am J Epidemiol. 2003;157:195-202.

35. Trogstad L, Magnus P, Moffett A, Stoltenberg C. The effect of recurrent miscarriage and infertility on the risk of pre-eclampsia. BJOG. 2009;116:108-13.

36. Saftlas AF, Levine RJ, Klebanoff MA, Martz KL, Ewell MG, Morris CD, et al. Abortion, changed paternity, and risk of preeclampsia in nulliparous women. Am J Epidemiol. 2003;157:1108-14.

37. Trogstad L, Magnus P, Skjaerven R, Stoltenberg C. Previous abortions and risk of pre-eclampsia. Int J Epidemiol. 2008;37:1333-40.

38. Hercus A, Dekker G, Leemaqz S. Primipaternity and birth interval; independent risk factors for preeclampsia. J Matern Fetal Neonatal Med. 2020;33:303-6.

39. Khong TY, Adema ED, Erwich JJ. On an anatomical basis for the increase in birth weight in second and subsequent born children. Placenta. 2003;24:348-53.

40. Darmochal-Kolarz D, Saito S, Rolinski J, Tabarkiewicz J, Kolarz B, Leszczynska-Gorzelak B, et al. Activated T lymphocytes in pre-eclampsia. Am J Reprod Immunol. 2007;58:39-45.

41. Marti JJ, Herrmann U. Immunogestosis: a new etiologic concept of "essential" EPH gestosis, with special consideration of the primigravid patient; preliminary report of a clinical study. Am J Obstet Gynecol. 1977;128:489-93.

42. Goecke C, Schwabe G. Vorschlag einer Stadien-Einteilung der Gestose. Zentralbl Gynaekol. 1965;87:1439. German.

43. Klonoff-Cohen HS, Savitz DA, Cefalo RC, McCann MF. An epidemiologic study of contraception and preeclampsia. JAMA. 1989;262:3143-7.

44. Einarsson JI, Sangi-Haghpeykar H, Gardner MO. Sperm exposure and development of preeclampsia. Am J Obstet Gynecol. 2003;188:1241-3.

45. Robillard PY, Hulsey TC, Perianin J, Janky E, Miri EH, Papiernik E. Association of pregnancy induced hypertension with duration of sexual cohabitation before conception. Lancet. 1994;344:973-5. 
46. Ness RB, Markovic N, Harger G, Day R. Barrier methods, length of preconception intercourse, and preeclampsia. Hypertens Pregnancy. 2004;23:227-35.

47. Robillard PY, Dekker GA, Hulsey TC. Evolutionary adaptations to pre-eclampsia/eclampsia in humans: low fecundability rate, loss of oestrus, prohibitions of incest and systematic polyandry. Am J Reprod Immunol. 2002;47:104-11.

48. Andraweera P, Roberts CT, Leemaqz S, McCowan L, Myers J, Kenny LC, et al. The duration of sexual relationship and its effects on adverse pregnancy outcomes. J Reprod Immunol. 2018;128:16-22.

49. Saftlas AF, Rubenstein L, Prater K, Harland KK, Field E, Triche EW. Cumulative exposure to paternal seminal fluid prior to conception and subsequent risk of preeclampsia. J Reprod Immunol. 2014;101-102:104-10.

50. Zhu D, Song Y, Ding Q, Duan C, Wu W, Xu J. Correlative research of the incidence of preeclampsia and sperm exposure. Arch Gynecol Obstet. 2021;304:695-701.

51. Koelman CA, Coumans AB, Nijman HW, Doxiadis II, Dekker GA, Claas FH. Correlation between oral sex and a low incidence of preeclampsia: a role for soluble HLA in seminal fluid? J Reprod Med. 2000;46:155-66.

52. Zavazava N, Kronke M. Soluble HLA class I molecules induce apoptosis in alloreactive cytotoxic T cells. Nat Med. 1996;2:1005-10.

53. Smith GN, Walker M, Tessier JL, Millar KG. Increased incidence of preeclampsia in women conceiving by intrauterine insemination with donor versus partner sperm for treatment of primary infertility. Am J Obstet Gynecol. 1997;177:455-8.

54. Wang JX, Knottnerus AM, Schuit G, Norman RJ, Chan A, Dekker GA. Surgically obtained sperm, and risk of gestational hypertension and pre-eclampsia. Lancet. 2002;359:673-4.

55. Tremellen KP, Seamark RF, Robertson SA. Seminal transforming growth factor beta1 stimulates granulocyte-macrophage colony-stimulating factor production and inflammatory cell recruitment in the murine uterus. Biol Reprod. 1998;58:1217-25.

56. Robertson SA. Transforming growth factor beta-a mediator of immune deviation in seminal plasma. J Reprod Immunol. 2002;57:109-28.

57. Than NG, Balogh A, Romero R, Kárpáti E, Erez O, Szilagyi A, et al. Placental protein 13 (PP13)—a placental immunoregulatory galectin protecting pregnancy. Front Immunol. 2014;5:348.

58. Schenker JG, Ezra Y. Complications of assisted reproductive techniques. Fertil Steril. 1994;61:411-22.

59. Need JA, Bell B, Meffin E, Jones WR. Pre-eclampsia in pregnancies from donor inseminations. J Reprod Immunol. 1983;5:329-38.

60. Grefenstette I, Royere D, Barthelemy Cl, Tharanne MJ, Lansac J. Outcome of 470 pregnancies after artificial insemination with frozen sperm. J Gynecol Obstet Biol Reprod (Paris). 1990;19:737-44. French.

61. Serhal PF, Craft I. Immune basis for pre-eclampsia: evidence from oocyte recipients. Lancet. 1987;2:744.

62. Serhal PF, Craft IL. Oocyte donation in 61 patients. Lancet. 1989;1:1185-7.

63. Salha O, Sharma V, Dada T, Nugent D, Rutherford AJ, Tomlinson AJ, et al. The influence of donated gametes on the incidence of hypertensive disorders of pregnancy. Hum Reprod. 1999;14:2268-73.

64. Blazquez A, García D, Vassena R, Figueras F, Rodriguez A. Risk of preeclampsia in pregnancies resulting from double gamete donation and from oocyte donation alone. Pregnancy Hypertens. 2018;13:133-7.

65. Moreno-Sepulveda J, Checa MA. Risk of adverse perinatal outcomes after oocyte donation: a systematic review and meta-analysis. J Assist Reprod Genet. 2019;36:2017-37.

66. Robillard PY, Dekker G, Chaouat G, Elliot MG, Scioscia M. High incidence of early onset preeclampsia is probably the rule and not the exception worldwide. 20th anniversary of the reunion workshop. A summary. J Reprod Immunol. 2019;133:30-6.

67. Dadelszen von P, Ornstein MP, Bull SB, Logan AG , Koren G, Magee LA. Fall in mean arterial pressure and fetal growth restriction in pregnancy hypertension: a meta-analysis. Lancet. 2000;355:87-92. 
68. Haig D. Genetic conflicts in human pregnancy. Q Rev Biol. 1993;68:495-532.

69. Kenny LC, Kell DB. Immunological tolerance, pregnancy, and preeclampsia: the roles of semen microbes and the father. Front Med (Lausanne). 2018;4:239. 